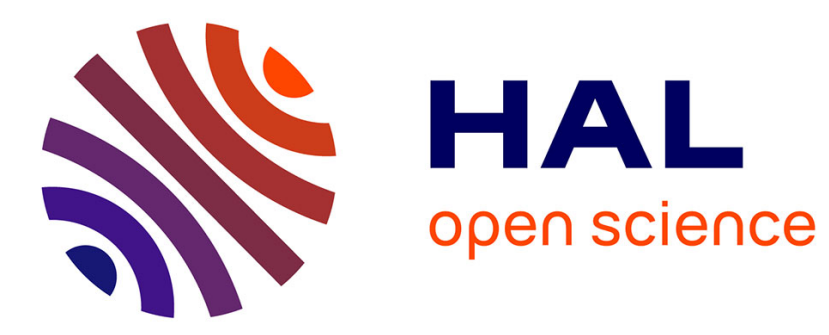

\title{
Alphabets, rewriting trails and periodic representations in algebraic bases
}

Denys Dutykh, Jean-Louis Verger-Gaugry

\section{To cite this version:}

Denys Dutykh, Jean-Louis Verger-Gaugry. Alphabets, rewriting trails and periodic representations in algebraic bases. Research in Number Theory, 2021, 7 (64). hal-03090468v3

\section{HAL Id: hal-03090468 \\ https://hal.science/hal-03090468v3}

Submitted on 29 Sep 2021

HAL is a multi-disciplinary open access archive for the deposit and dissemination of scientific research documents, whether they are published or not. The documents may come from teaching and research institutions in France or abroad, or from public or private research centers.
L'archive ouverte pluridisciplinaire HAL, est destinée au dépôt et à la diffusion de documents scientifiques de niveau recherche, publiés ou non, émanant des établissements d'enseignement et de recherche français ou étrangers, des laboratoires publics ou privés.

\section{(이) $\$$}

Distributed under a Creative Commons Attribution - NonCommercial - NoDerivatives| 4.0 


\title{
ALPHABETS, REWRITING TRAILS AND PERIODIC REPRESENTATIONS IN ALGEBRAIC BASES
}

\author{
DENYS DUTYKH $\dagger$ AND JEAN-LOUIS VERGER-GAUGRY
}

\begin{abstract}
For $\beta>1$ a real algebraic integer (the base), the finite alphabets $\mathscr{A} \subset \mathbb{Z}$ which realize the identity $\mathbb{Q}(\beta)=\operatorname{Per}_{\mathscr{A}}(\beta)$, where $\operatorname{Per}_{\mathscr{A}}(\beta)$ is the set of complex numbers which are $(\beta, \mathscr{A})$-eventually periodic representations, are investigated. Comparing with the greedy algorithm, minimal and natural alphabets are defined. The natural alphabets are shown to be correlated to the asymptotics of the Pierce numbers of the base $\beta$ and Lehmer's problem. The notion of rewriting trail is introduced to construct intermediate alphabets associated with small polynomial values of the base. Consequences on the representations of neighbourhoods of the origin in $\mathbb{Q}(\beta)$, generalizing Schmidt's theorem related to Pisot numbers, are investigated. Applications to Galois conjugation are given for convergent sequences of bases $\gamma_{s}:=\gamma_{n, m_{1}, \ldots, m_{s}}$ such that $\gamma_{s}^{-1}$ is the unique root in $(0,1)$ of an almost Newman polynomial of the type $-1+x+x^{n}+x^{m_{1}}+\ldots+x^{m_{s}}, n \geq 3, s \geq 1, m_{1}-n \geq n-1$, $m_{q+1}-m_{q} \geq n-1$ for all $q \geq 1$. For $\beta>1$ a reciprocal algebraic integer close to one, the poles of modulus $<1$ of the dynamical zeta function of the $\beta$-shift $\zeta_{\beta}(z)$ are shown, under some assumptions, to be zeroes of the minimal polynomial of $\beta$.
\end{abstract}

Keywords: alphabet, periodic representation, Pierce number, Galois conjugate, betashift, dynamical zeta function.

2020 Mathematics Subject Classification: 11A63, 11A67, 11B83, 11K16, 11R04, 11R06.

\section{CONTENTS}

1. Introduction 2

2. Natural alphabets in $(\beta, \mathscr{A})$-periodic representations of $\mathbb{Q}(\beta)$

2.1. Pierce numbers of the base and integer polynomials with a dominant coefficient 5

2.2. Natural alphabets for a convergent sequence of Pisot numbers 8

3. Small heights and eventually periodic representations of polynomial values of the base

3.1. Rewriting trails, intermediate alphabets - Proof of Theorem 1.4

3.2. Application to Galois conjugation: convergence and eventually periodic representations along a sequence of almost Newman polynomials

3.3. Natural and intermediate alphabets along sequences of almost Newman polynomials: examples 


\section{INTRODUCTION}

For a general complex number $\beta \in \mathbb{C},|\beta|>1$, and a finite alphabet $\mathscr{A} \subset \mathbb{C}$, we define the $(\beta, \mathscr{A})$-representations as expressions of the form $\sum_{k \geq-L} a_{k} \beta^{-k}, a_{k} \in \mathscr{A}$, for some integer $L \in \mathbb{Z}$. They are Laurent series of $1 / \beta$. We define

$$
\operatorname{Per}_{\mathscr{A}}(\beta):=\{x \in \mathbb{C} \mid x \text { has an eventually periodic }(\beta, \mathscr{A}) \text {-representation }\} .
$$

In this note attention is focused on the complex numbers $\beta$ which are real algebraic integers $>1$, close to 1 , assuming that $\beta$ has no conjugate on the unit circle, and on the alphabets $\mathscr{A} \subset \mathbb{Z}$, depending upon $\beta$, involved in the identity:

$$
\mathbb{Q}(\beta)=\operatorname{Per}_{\mathscr{A}}(\beta) .
$$

We write $\mathbb{Q}$ for the set of rational numbers, $\mathbb{Q}(\beta)$ for the smallest sub-field of $\mathbb{C}$ containing $\beta$. Indeed, such an identity always holds by the following theorem.

Theorem 1.1 (Kala -Vávra [13]). Let $\beta \in \mathbb{C}$ be an algebraic number of degree $d,|\beta|>1$, and let $a_{d} x^{d}-a_{d-1} x^{d-1}-\ldots-a_{1} x-a_{0} \in \mathbb{Z}[x]$ be its minimal polynomial. Suppose that $\left|\beta^{\prime}\right| \neq 1$ for any conjugate $\beta^{\prime}$ of $\beta$. Then there exists a finite alphabet $\mathscr{A} \subset \mathbb{Z}$ such that

$$
\mathbb{Q}(\beta)=\operatorname{Per}_{\mathscr{A}}(\beta) \text {. }
$$

Theorem 1.1 is a generalization of a previous theorem of Baker, Masáková, Pelantová and Vávra [1] in which $1 / a_{d}$ was assumed to belong to $\mathbb{Z}\left[\beta, \beta^{-1}\right]$, an assumption removed in [13].

In Section 2 we revisit the construction of an alphabet $\mathscr{A} \subset \mathbb{Z}$, symmetrical with respect to the origin, which allows (1.0.1) to hold, given in [12]. We show that the size of this alphabet is correlated to the Pierce numbers $\Delta_{N}(\beta)$ of $\beta$. The numerical explosion of $\Delta_{N}(\beta)$ with $N$ has been investigated in [7]. Pierce numbers play an important role in the Mahler measure of $\beta$ and the search of big prime numbers (Lehmer [19], Einsiedler, Everest and Ward [7]). The alphabet constructed by this means is called the natural alphabet realizing (1.0.1). We denote it by $\mathscr{A}_{\beta}^{(\text {nat })}$. It has no reason to be the smallest one realizing (1.0.1).

Remark 1.2. Denote by

$$
\widehat{\mathscr{A}}:=\{\{-m,-m+1, \ldots,-1,0,+1, \ldots, m-1, m\} \mid m \in \mathbb{N} \backslash\{0\}\}
$$

the set of symmetrical alphabets with digits in $\mathbb{Z}$. It is totally ordered by inclusion. If $\mathscr{A}_{1}=\left\{-m_{1}, \ldots, 0, \ldots, m_{1}\right\}, \mathscr{A}_{2}=\left\{-m_{2}, \ldots, 0, \ldots, m_{2}\right\}$, are two elements of $\widehat{\mathscr{A}}$, then

$$
\mathscr{A}_{1} \subset \mathscr{A}_{2} \quad \text { if and only if } \quad m_{1} \leq m_{2} \text {. }
$$

The explicit construction of the map $\beta \rightarrow \mathscr{A}_{\beta}^{(\text {nat })} \in \widehat{\mathscr{A}}$, as in Section 2, proves the existence of at least one alphabet say $\mathscr{A}_{\beta} \in \widehat{\mathscr{A}}$ realizing (1.0.1), included (a priori not necessarily strictly) in $\mathscr{A}_{\beta}^{(\text {nat })}$. This justifies the terminology "natural" for $\mathscr{A}_{\beta}^{(\text {nat })}$. Let us note that, if a finite alphabet $\mathscr{A}_{\beta} \in \widehat{\mathscr{A}}$ realizes (1), then any of its finite supersets does that, and could be bigger than $\mathscr{A}_{\beta}^{(\text {nat })}$. Therefore there is interest in characterizing the symmetrical alphabets $\mathscr{A}_{\beta} \subset \mathscr{A}_{\beta}^{(\text {nat })}$ which realize (1.0.1). Because of the total ordering of $\widehat{\mathscr{A}}$, among all of them, there is an unique smallest element, say $\mathscr{A}_{\text {mini }}$. We have 


$$
\{-1,0,+1\} \subset \ldots \subset \mathscr{A}_{\text {mini }} \subset \ldots \subset \mathscr{A}_{\beta} \subset \ldots \subset \mathscr{A}_{\beta}^{(\text {nat })} \subset \ldots
$$

Problem: For $\beta$ any real algebraic integer $>1$ such that $\beta$ has no conjugate on the unit circle, what is the minimal symmetrical alphabet $\mathscr{A} \subset \mathbb{Z}, \mathscr{A} \in \widehat{\mathscr{A}}$, realizing (1.0.1)?

The minimal alphabet $\mathscr{A}_{\text {mini }}$ a priori depends upon $\beta$. Intermediate alphabets between $\mathscr{A}_{\text {mini }}$ and $\mathscr{A}_{\beta}^{(\text {nat })}$ realizing (1.0.1) are investigated by introducing rewriting trails in Section 3.

If $\beta$ is a Pisot number the problem is solved by the following theorem [23], with the minimal alphabet $\mathscr{A}=\{-1,0,+1\}$ (independent of $\beta$ ). The set $\operatorname{Per}_{\{0,1\}}(\beta)$ is the set of (eventually) periodic points for the $\beta$-transformation $T_{\beta}: x \rightarrow\{\beta x\}$ on $[0,1)$, i.e. for the set of points whose orbits under $T_{\beta}$, are finite. The $(\beta,\{-1,0,1\})$-eventually periodic representations of the elements $x \in \mathbb{Q}(\beta) \cap(-1,+1)$ are the Rényi expansions, equivalently they are constructed from the greedy algorithm. Then all the elements of $\mathbb{Q}(\beta)$ have eventually periodic representations.

Theorem 1.3 (K. Schmidt [23]). Let $\beta>1$ be a real number.

(1) If $\mathbb{Q} \cap[0,1) \subset \operatorname{Per}_{\{0,1\}}(\beta)$, then $\beta$ is either a Pisot or a Salem number.

(2) If $\beta$ is a Pisot number, then $\operatorname{Per}_{\{0,1\}}(\beta)=\mathbb{Q}(\beta) \cap[0,1]$.

If $\beta$ is a Pisot number and $x \in[-1,0] \cap \mathbb{Q}(\beta)$, then $-x$ admits an eventually periodic representation in base $\beta$, with digits in $\{-1,0\}$, which is the opposite of the one of $|x|$, so that any $x \in[-1,+1]$, hence any $x \in \mathbb{Q}(\beta)$, has an eventually periodic representation with digits in the symmetric alphabet $\{-1,0,+1\}$. By comparison, the natural alphabets $\mathscr{A}_{\beta_{k}}^{(\text {nat })}$ associated to the Pisot numbers $\beta_{k}$ belonging to an increasing sequence tending to $(1+\sqrt{5}) / 2$, calculated by means of Proposition 2.1, are studied in Section 2.2.

Daróczy and Kátai [4], and later Thurston [27], have proved that for any non-real $\beta \in$ $\mathbb{C},|\beta|>1$, there exists a finite alphabet $\mathscr{A} \subset \mathbb{C}$ such that every $x \in \mathbb{C}$ has a $(\beta, \mathscr{A})$ representation. The search for periodic representations in radix systems goes back to Kovács [15] and to Kovács and Környei [16] (see also Pethő [22]). For the Rényi-Parry numeration system in base $\beta>1$, the idea of the enlargement of the alphabet to obtain the eventual periodicity for the representations of the elements of the number field $\mathbb{Q}(\beta)$ is recurrent.

Theorem 1.4 extends Schmidt's Theorem 1.3 to the representations of the elements of $\mathbb{Q}(\gamma) \cap \mathscr{V}$ where $\mathscr{V}$ is a neighbourhood of the origin, and $\gamma>1$ an algebraic integer, root of a polynomial with coefficients in $\{-1,0,1\}$, having no conjugate on the unit circle. In Section 3.1 we introduce the notion of rewriting trail. We show that intermediate alphabets, between the minimal and the natural ones, are produced by rewriting trails. The proof of Theorem 1.4 is based on rewriting trails, and makes use of Kala - Vavra's Theorem 1.1.

Theorem 1.4. Let $\gamma>1$ be an algebraic integer, root of a polynomial $S_{\gamma}(X)=X^{s}-$ $\sum_{i=0}^{s-1} t_{s-i} X^{i}$, with $s \geq 1, t_{i} \in \mathbb{Z},\left|t_{i}\right| \leq 1$, not necessarily irreducible, such that $\left|\gamma^{\prime}\right| \neq 1$ for any conjugate $\gamma^{\prime}$ of $\gamma$.

Let $P(X)=1+a_{1} X+a_{2} X^{2}+\ldots+a_{d-1} X^{d-1}+a_{d} X^{d} \in \mathbb{Z}[X], d=\operatorname{deg} P \geq 1$, be an integer polynomial. Denote by $H=\max _{i=1, \ldots, d}\left|a_{i}\right|$ the height of $P$. 
Let $0<\eta<1$ and suppose $0 \neq|P(\gamma)|<\eta$. Then the polynomial value $P(\gamma) \in \mathbb{Q}(\gamma)$ admits at least one eventually periodic representation

$$
P(\gamma)=R\left(\gamma^{-1}\right)+\frac{1}{\gamma^{L}} \sum_{j=0}^{\infty} \frac{1}{\gamma^{j r}} T\left(\gamma^{-1}\right) \quad \in \operatorname{Per}_{\mathscr{A}}(\gamma)
$$

with

(i) alphabet $\mathscr{A}=\{-m, \ldots,+m\} \subset \mathbb{Z}, m=\left\lceil 2\left(\left(2^{d}-1\right) H+2^{d}\right) / 3\right\rceil$, independent of $s$ and $\gamma$,

(ii) $R(X) \in \mathscr{A}[X], \operatorname{deg} R \leq s-1, T(X) \in \mathscr{A}[X], \operatorname{deg} T \leq s-1$, and $L$ and $r$ being some integers satisfying $L>\operatorname{deg} R, r>\operatorname{deg} T$,

(iii) preperiod

$$
R\left(\gamma^{-1}\right)=\frac{a_{w}}{\gamma^{w}}+\frac{a_{w+1}}{\gamma^{w+1}}+\ldots+\frac{a_{w+s-1}}{\gamma^{w+s-1}}, \quad a_{j} \in \mathscr{A}, j=w, \ldots, w+s-1, a_{w} \neq 0
$$

with $w \geq 1$ satisfying $\frac{\kappa_{\gamma, \mathscr{A}}}{\eta} \leq \gamma^{w-1}$ for some positive constant $\kappa_{\gamma, \mathscr{A}}$ depending upon $\gamma$ and $\mathscr{A}$.

Remark 1.5. In Theorem 1.4 the polynomial $S_{\gamma}(X)$ could have some zeroes of modulus one. For instance, if it is of the form $S_{\gamma}(X)=A(X) \times C(X)$ with $A(X)$ a product of cyclotomic polynomials and $C(X)$ the minimal polynomial of $\gamma$. The assumption that the conjugates $\gamma^{\prime}$ do not lie on the unit circle only concerns the zeroes of $C(X)$.

In Section 3.2 Theorem 1.4 is applied to the Galois conjugation of eventually periodic representations of polynomial values of the base $\gamma$ for $\gamma$ runing over a sequence of real algebraic integers converging towards a reciprocal algebraic integer $\beta>1$. The consequences on the Galois conjugates of $\beta$ of modulus $<1$ are investigated in the context of automorphisms of complex numbers (Kestelman [14], Yales [30]); the absence of continuity of the $\mathbb{Q}$-automorphisms of conjugation is compensated in some sense by the eventual periodicity of the representations. Proposition 3.5 reports some consequences on the relations between the poles of the dynamical zeta function $\zeta_{\beta}(z)$ of the $\beta$-shift (see e.g. Solomyak [25]) and the zeroes of the minimal polynomial of $\beta$. Examples of natural alphabets related to sequences of polynomials of the class $\mathscr{B}$ are studied in Section 3.3, in terms of sequences of Mahler measures.

\section{Natural Alphabets in $(\beta, \mathscr{A})$-PERIOdiC REPRESEntations of $\mathbb{Q}(\beta)$}

Let $t \geq 1$. A polynomial $Q(X)=\sum_{i=0}^{d} a_{i} X^{i} \in \mathbb{Z}[X]$ is said to have a dominant coefficient, resp. to be a $t$-polynomial, if there exists an integer $j \in\{0,1, \ldots, d\}$ such that $\left|a_{j}\right|>\sum_{i=0, i \neq j}^{d}\left|a_{i}\right|$, resp. $\left|a_{j}\right|>t \sum_{i=0, i \neq j}^{d}\left|a_{i}\right|$. Let $\beta$ be an algebraic integer $>1$ having no conjugate on the unit circle. If the ideal $\left(P_{\beta}\right)=P_{\beta}(X) \mathbb{Z}[X]$ generated by the minimal polynomial $P_{\beta}(X)$ of $\beta$ contains a 1-polynomial $\sum_{i=0}^{d} a_{i} X^{i}$, of dominant coefficient $a_{j}$, then, by Proposition 5.1 in [12] and Theorem 25 in [1], the alphabet

$$
\{-m, \ldots, 0, \ldots, m\}, \quad \text { with } \quad m:=\left\lceil\frac{\left|a_{j}\right|-1}{2}\right\rceil+\sum_{i=0, i \neq j}^{d}\left|a_{i}\right|,
$$

satisfies (1.0.1). Here \lceil\rceil denotes the upper integer part. In Section 2.1 we recall an effective construction of such a 1-polynomial in $\left(P_{\beta}\right)$. The proof of Proposition 2.1 is reproduced 
from [12] to fix the notations. The way it is obtained comes from a necessarily finite number of successive iterations of the companion matrix of $P_{\beta}$.

\subsection{Pierce numbers of the base and integer polynomials with a dominant coefficient.}

Proposition 2.1. Let $\alpha$ be an algebraic integer, of degree $d,|\alpha|>1$, of minimal polynomial $P_{\alpha}(X)=\prod_{j=1}^{d}\left(X-\alpha_{(j)}\right)$, with $\alpha=\alpha_{(1)}$ and $\left|\alpha_{(j)}\right| \neq 1$ for $j=2,3, \ldots, d$. Denote by $j_{0}$ the number of conjugates $\alpha_{(j)}$ of $\alpha$ which have a modulus $>1$. Then, for any $t \geq 1$, there exist an integer $N$ and a polynomial

$$
Q(X)=X^{d N}+a_{1} X^{(d-1) N}+a_{2} X^{(d-2) N}+\ldots+a_{d-1} X^{N}+a_{d} \quad \in \mathbb{Z}[X]
$$

such that $Q(\alpha)=0$, setting $a_{0}=1$, with

$$
\left|a_{j_{0}}\right|>t \sum_{i \in\{0,1,2, \ldots, d\} \backslash\left\{j_{0}\right\}}\left|a_{i}\right| .
$$

Proof. We have $j_{0} \geq 1$. The minimal polynomial

$$
P_{\alpha}(X)=\prod_{j=1}^{d}\left(X-\alpha_{(j)}\right)=X^{d}+g_{1} X^{d-1}+g_{2} X^{d-2}+\ldots+g_{d-1} X+g_{d} \in \mathbb{Z}[X]
$$

can be written as the characteristic polynomial of $\alpha$, from the companion matrix [18]

$$
H=\left(\begin{array}{cccccc}
0 & 0 & & \ldots & 0 & -g_{d} \\
1 & 0 & 0 & \ldots & 0 & -g_{d-1} \\
0 & 1 & 0 & \ldots & 0 & -g_{d-2} \\
\vdots & & & & & \\
\vdots & & & 1 & 0 & -g_{2} \\
0 & \ldots & 0 & & 1 & -g_{1}
\end{array}\right)
$$

We have: $\operatorname{det}\left(H-X \mathrm{I}_{d}\right)=(-1)^{d} P_{\alpha}(X)$, where $\mathrm{I}_{d}$ is the identity matrix. The eigenvalues of $H$ are the zeroes of $P_{\alpha}(X)$. For $n \geq 2$ let us define

$$
P_{\alpha, n}(X):=(-1)^{d} \operatorname{det}\left(H^{n}-X \mathrm{I}_{d}\right) \in \mathbb{Z}[X] .
$$

The polynomial $P_{\alpha, n}(X)$, of degree $d$, has integer coefficients

$$
P_{\alpha, n}(X)=\prod_{j=1}^{d}\left(X-\alpha_{(j)}^{n}\right)=X^{d}+g_{1}(n) X^{d-1}+g_{2}(n) X^{d-2}+\ldots+g_{d-1}(n) X+g_{d}(n) .
$$

We set: $g_{j}=g_{j}(1)$ for $j=1,2, \ldots, n$ and $g_{0}=g_{0}(n)=1$ for $n \geq 1$. The coefficients $g_{j}(n)$ are related to the symmetric functions of the roots. Without loss of generality, let us assume:

$$
\left|\alpha_{(1)}\right| \geq\left|\alpha_{(2)}\right| \geq \ldots \geq\left|\alpha_{\left(j_{0}\right)}\right|>\left|\alpha_{\left(j_{0}+1\right)}\right| \geq \ldots \geq\left|\alpha_{(d)}\right|
$$

where $j_{0}:=\max \left\{i: 1<\left|\alpha_{(i)}\right|\right\}$. The choice of $j_{0}$ guarantees

$$
\left|\frac{\alpha_{\left(i_{1}\right)} \alpha_{\left(i_{2}\right)} \ldots \alpha_{\left(i_{r}\right)}}{\alpha_{(1)} \alpha_{(2)} \ldots \alpha_{\left(j_{0}\right)}}\right|<1
$$


for any subset $\left\{i_{1}, i_{2}, \ldots, i_{r}\right\} \subset\{1,2, \ldots, d\}$ and $\left\{i_{1}, i_{2}, \ldots, i_{r}\right\} \neq\left\{1,2, \ldots, j_{0}\right\}$. Then, for all choices of $\left\{i_{1}, i_{2}, \ldots, i_{r}\right\} \neq\left\{1,2, \ldots, j_{0}\right\}$, we have:

$$
\lim _{n \rightarrow \infty} \frac{\alpha_{\left(i_{1}\right)}^{n} \alpha_{\left(i_{2}\right)}^{n} \ldots \alpha_{\left(i_{r}\right)}^{n}}{\alpha_{(1)}^{n} \alpha_{(2)}^{n} \ldots \alpha_{\left(j_{0}\right)}^{n}}=0
$$

Now, for all $n \geq 1,1 \leq j \leq d$, we have:

$$
g_{j}(n)=\sum_{\left\{i_{1}, i_{2}, \ldots, i_{j}\right\} \in S_{j}} \alpha_{\left(i_{1}\right)}^{n} \alpha_{\left(i_{2}\right)}^{n} \ldots \alpha_{\left(i_{j}\right)}^{n}
$$

where $S_{j}=\{\mathscr{P} \subset\{1,2, \ldots, d\}: \# \mathscr{P}=j\}$ is the set of all subsets of $\{1,2, \ldots, d\}$ with cardinality $j$. Since

$$
\lim _{n \rightarrow \infty} \frac{g_{j}(n)}{\alpha_{(1)}^{n} \alpha_{(2)}^{n} \ldots \alpha_{\left(j_{0}\right)}^{n}}=\left\{\begin{array}{c}
0 \\
(-1)^{j}
\end{array} \text { for all } j=\begin{array}{c}
1,2, \ldots, d \text { and } j \neq j_{0} \\
\text { for } j=j_{0}
\end{array},\right.
$$

we deduce that, for any $t>0$, there exists an integer $N=N(t)$ such that

$$
\frac{\left|g_{j_{0}}(N)\right|}{\left|\alpha_{(1)}^{N} \alpha_{(2)}^{N} \ldots \alpha_{\left(j_{0}\right)}^{N}\right|}>t \sum_{j \in\{0,1,2, \ldots, d\}, j \neq j_{0}} \frac{\left|g_{j}(N)\right|}{\left|\alpha_{(1)}^{N} \alpha_{(2)}^{N} \ldots \alpha_{\left(j_{0}\right)}^{N}\right|}
$$

equivalently

$$
\left|g_{j_{0}}(N)\right|>t \sum_{j \in\{0,1,2, \ldots, d\}, j \neq j_{0}}\left|g_{j}(N)\right|
$$

This inequality gives the result (2.1.1), with $Q(X)=P_{\alpha, N}\left(X^{N}\right)$.

Definition 2.2. The smallest integer $N=N(t)$ for which (2.1.2) is satisfied is called the dominance index of $P_{\alpha}$ (or of $\left.\alpha\right)$ for the value $t \geq 1$. For $t=1, N(1)$ is called the dominance index of $P_{\alpha}$ (or of $\left.\alpha\right)$.

Definition 2.3. Let $\alpha>1$ be a real algebraic integer. With the same notations as in Proposition 2.1 and its proof, the alphabet $:=\{-m, \ldots, 0, \ldots, m\}$, with

$$
m:=\left\lceil\frac{\left|g_{j_{0}}(N)\right|-1}{2}\right\rceil+\sum_{j=0, j \neq j_{0}}^{d}\left|g_{j}(N)\right|
$$

and $N$ the dominance index of $\alpha$, is called the natural alphabet of $\alpha$, and denoted by $\mathscr{A}_{\alpha}^{(\text {nat })}$.

For $\alpha>1$ any real algebraic integer, the existence of the natural alphabet $\mathscr{A}_{\alpha}^{(\text {nat })}$ implies that $\alpha$ satisfies the weak representation of zero property, or, for short, $\alpha$ is WRZ, in the terminology of [12]. Then, in the Sections 4 and 5 in [12], Frougny, Pelantova and Svobodova provide a parallel algorithm "Algorithm II" which gives access to (1.0.1).

Proposition 2.4. Let $\alpha>1$ be a real algebraic integer. With the same notations as in Proposition 2.1 and its proof, with $N=N(t)$ the smallest value which satisfies (2.1.2), we have:

$$
\left|g_{j_{0}}(N)\right|>\frac{t}{1+t} \Delta_{N}(\alpha)
$$


where $\left|P_{\alpha, N}(1)\right|=\Delta_{N}(\alpha)=\left|\prod_{j=1}^{d}\left(1-\alpha_{(j)}^{N}\right)\right|$ is the $N$-th Pierce number of $\alpha$, and the natural alphabet $\mathscr{A}_{\alpha}^{(\text {nat })}=\{-m, \ldots, 0, \ldots, m\}$ is such that

$$
m \geq\left\lceil 2^{-1}\left(2^{-1} \Delta_{N}(\alpha)-1\right)\right\rceil .
$$

Proof. In the continuation of (2.1.2), we have

$$
\begin{gathered}
\sum_{j \in\{0,1,2, \ldots, d\}, j \neq j_{0}}\left|g_{j}(N)\right| \geq\left|\sum_{j=0}^{d} g_{j}(N)-g_{j_{0}}(N)\right|=\left|P_{\alpha, N}(1)-g_{j_{0}}(N)\right| \\
\geq|| P_{\alpha, N}(1)|-| g_{j_{0}}(N)|| \geq \Delta_{N}(\alpha)-\left|g_{j_{0}}(N)\right| .
\end{gathered}
$$

Therefore

$$
\left|g_{j_{0}}(N)\right|>t \Delta_{N}(\alpha)-t\left|g_{j_{0}}(N)\right|
$$

equivalently (2.1.3). We now take $t=1, N=N(1)$ the dominance index of $\alpha$, and apply Proposition 5.1 in [12] and Theorem 25 in [1].

Remark 2.5. To each polynomial of the form $P_{\alpha}$ as in Proposition 2.1 there is an associated endomorphism $\mathrm{T}_{P_{\alpha}}$ of the $d$-torus, given by the natural action of the companion matrix of $P_{\alpha} . \mathrm{T}_{P_{\alpha}}$ is an ergodic transformation with respect to Lebesgue measure, and $\Delta_{N}\left(P_{\alpha}\right)$ is the number of points of period $\mathrm{N}$ under $\mathrm{T}_{P_{\alpha}}$ [8]. The Mahler measure $\mathrm{M}(\alpha)=\prod_{i=1}^{d} \max \left\{1,\left|\alpha_{(i)}\right|\right\}$ of $\alpha$ is related to the dynamical properties of the corresponding toral endomorphism. The condition of having no root on the unit circle implies expansiveness of $\mathrm{T}_{P_{\alpha}}$ as a topological dynamical system. The topological entropy of $\mathrm{T}_{P_{\alpha}}$ is equal to $\log \mathrm{M}(\alpha)[20]$.

Remark 2.6. The link between the natural alphabet $\mathscr{A}_{\beta}^{(\text {nat })}$ and the Mahler measure $\mathrm{M}(\alpha)$ of the base of numeration $\alpha$ naturally comes from Proposition 2.1 where $j_{0}$ counts the number of roots outside the closed unit disk. It can be estimated roughly as follows: first the $N$-th Pierce number of $\alpha$ is

$$
\Delta_{N}(\alpha)=\frac{\Delta_{N}(\alpha)}{\Delta_{N-1}(\alpha)} \times \frac{\Delta_{N-1}(\alpha)}{\Delta_{N-2}(\alpha)} \times \ldots \times \frac{\Delta_{2}(\alpha)}{\Delta_{1}(\alpha)} \Delta_{1}(\alpha),
$$

with $\Delta_{1}(\alpha)=\left|P_{\alpha}(1)\right|$. From Lehmer [19],

$$
\mathrm{M}(\alpha)=\lim _{q \rightarrow \infty} \frac{\Delta_{q+1}(\alpha)}{\Delta_{q}(\alpha)},
$$

we deduce, without taking into account the type of convergence towards $\mathrm{M}(\alpha)$, as a rough estimate for the lower bound,

$$
\left|g_{j_{0}}(N)\right|>\frac{1}{2} \mathrm{M}(\alpha)^{N-1}\left|P_{\alpha}(1)\right|
$$

and the approximate lower bound $\left\lceil 2^{-1}\left(2^{-1} \mathrm{M}(\alpha)^{N-1}\left|P_{\alpha}(1)\right|-1\right)\right\rceil$ for $m$. Let us note that the sequence $\left(\Delta_{n}(\alpha)\right)_{n}$ is fairly chaotic, as the sequence of the Pierce numbers of $\alpha$, from the heuristics of Einsiedler, Everest and Ward [7]. 


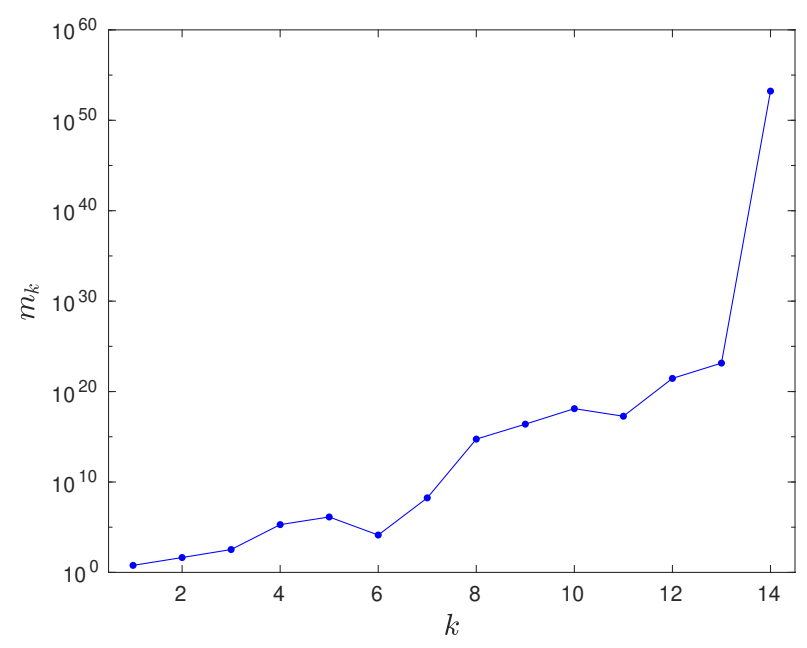

FIGURE 1. Natural alphabets of the Pisot numbers $\beta_{k}$.

2.2. Natural alphabets for a convergent sequence of Pisot numbers. In this paragraph we examplify the hugeness of the natural alphabets for a sequence of Pisot numbers. [3])

Let us consider the sequence of irreducible integer polynomials (from Theorem 7.2.1 in

$$
P_{2 k}(z)=\left(1-z^{2 k}\left(1+z-z^{2}\right)\right) /(1-z), \quad k \geq 1 .
$$

The dominant root of $P_{2 k}(z)$ is denoted $\beta_{k}>1$. All the other roots have a modulus $<1$. For all $k \geq 1$, we have: $\beta_{k}<(1+\sqrt{5}) / 2$. The sequence $\left(\beta_{k}\right)_{k \geq 1}$ is an increasing sequence of Pisot numbers, with limit: $\lim _{k \rightarrow \infty} \beta_{k}=\frac{1+\sqrt{5}}{2}$. For $k=1$ we have $P_{2}(z)=z^{3}-z-1$ and $\beta_{1}$ is the smallest Pisot number. Let $\tau=\beta_{\infty}=(1+\sqrt{5}) / 2$. It is the dominant root of the trinomial $z^{2}-z-1$.

The dominance index of $\tau$ is 3 , and the natural alphabet $\mathscr{A}_{\tau}^{(\text {nat })}$ is $=\{-3, \ldots,+3\}$. The growth rate of the natural alphabet $\mathscr{A}_{\beta_{k}}^{(\text {nat })}=\left\{-m_{k}, \ldots, m_{k}\right\}$ is represented as a function of $k$ in Figure 1.

\section{SMALl HeightS AND EVENTUALLY PERIODIC REPRESENTATIONS OF POLYNOMIAL VALUES OF THE BASE}

3.1. Rewriting trails, intermediate alphabets - Proof of Theorem 1.4. Denote by $S_{\gamma}^{*}(X)=$ $X^{s} S_{\gamma}(1 / X)=1-t_{1} X-t_{2} X^{2}-\ldots-t_{s-1} X^{s-1}-t_{s} X^{s}$ the reciprocal polynomial of $S_{\gamma}(X)=$ $X^{s}-\sum_{i=0}^{s-1} t_{s-i} X^{i}$. The coefficients $t_{i}$ are in $\{-1,0,+1\}$. The algebraic integer $\gamma$ is called the base and $S_{\gamma}^{*}\left(\gamma^{-1}\right)=0$.

We want to express $P(\gamma)$ as a $(\gamma, \mathscr{A})$ - eventually periodic representation with a certain alphabet $\mathscr{A}$ to be defined. This objective means that, first, we have to express $P(\gamma)$ as a Laurent series of $1 / \gamma$.

We now introduce a construction, that we call "rewriting trail from " $S_{\gamma}^{*}$ " to " $P$ ", at $\gamma^{-1}$, to reach this objective, and which will allow us to show that a symmetrical alphabet 
$\mathscr{A}=\{-m, \ldots, 0, \ldots, m\}$ can be defined and is such that $m$ depends upon $H$ and $\operatorname{deg}(P)$, independently of $s$ and $\gamma$.

The starting point is the identity $1=1$, to which we add $0=-S_{\gamma}^{*}\left(\gamma^{-1}\right)$ in the right hand side. Then we define a rewriting trail from

$$
1=1-S_{\gamma}^{*}\left(\gamma^{-1}\right)=t_{1} \gamma^{-1}+t_{2} \gamma^{-2}+\ldots+t_{s-1} \gamma^{-(s-1)}+t_{s} \gamma^{-s}
$$

to

$$
-a_{1} \gamma^{-1}-a_{2} \gamma^{-2}+\ldots-a_{d-1} \gamma^{-(d-1)}-a_{d} \gamma^{-d}=1-P\left(\gamma^{-1}\right) .
$$

A rewriting trail will be a sequence of integer polynomials, whose role will consist in "restoring" the coefficients of $1-P\left(\gamma^{-1}\right)$ one after the other, from the left, by adding " 0 " conveniently at each step to both sides of (3.1.1). At the first step we add $0=\left(-a_{1}-\right.$ $\left.t_{1}\right) \gamma^{-1} S_{\gamma}^{*}\left(\gamma^{-1}\right)$; and we obtain

$$
\begin{gathered}
1=-a_{1} \gamma^{-1} \\
+\left(-\left(-a_{1}-t_{1}\right) t_{1}+t_{2}\right) \gamma^{-2}+\left(-\left(-a_{1}-t_{1}\right) t_{2}+t_{3}\right) \gamma^{-3}+\ldots
\end{gathered}
$$

so that the height of the polynomial

$$
\left(-\left(-a_{1}-t_{1}\right) t_{1}+t_{2}\right) X^{2}+\left(-\left(-a_{1}-t_{1}\right) t_{2}+t_{3}\right) X^{3}+\ldots
$$

is $\leq H+2$. At the second step we add $0=\left(-a_{2}-\left(-\left(-a_{1}-t_{1}\right) t_{1}+t_{2}\right)\right) \gamma^{-2} S_{\gamma}^{*}\left(\gamma^{-1}\right)$. Then we obtain

$$
\begin{gathered}
1=-a_{1} \gamma^{-1}-a_{2} \gamma^{-2} \\
+\left[\left(-a_{2}-\left(-\left(-a_{1}-t_{1}\right) t_{1}+t_{2}\right)\right) t_{1}+\left(-\left(-a_{1}-t_{1}\right) t_{2}+t_{3}\right)\right] \gamma^{-3}+\ldots
\end{gathered}
$$

where the height of the polynomial

$$
\left[\left(-a_{2}-\left(-\left(-a_{1}-t_{1}\right) t_{1}+t_{2}\right)\right) t_{1}+\left(-\left(-a_{1}-t_{1}\right) t_{2}+t_{3}\right)\right] X^{3}+\ldots
$$

is $\leq H+(H+2)+(H+2)=3 H+4$. Iterating this process $d$ times we obtain

$$
\begin{gathered}
1=-a_{1} \gamma^{-1}-a_{2} \gamma^{-2}-\ldots-a_{d} \gamma^{-d} \\
+ \text { polynomial remainder in } \gamma^{-1}
\end{gathered}
$$

Denote by $V\left(\gamma^{-1}\right)$ this polynomial remainder in $\gamma^{-1}$, for some $V(X) \in \mathbb{Z}[X]$, and $X$ specializing in $\gamma^{-1}$. If we denote the upper bound of the height of the polynomial remainder $V(X)$, at step $q$, by $\lambda_{q} H+v_{q}$, we readily deduce: $v_{q}=2^{q}$, and $\lambda_{q+1}=2 \lambda_{q}+1, q \geq 1$, with $\lambda_{1}=1$; then $\lambda_{q}=2^{q}-1$.

To summarize, we obtain a sequence $\left(A_{q}^{\prime}(X)\right)_{q \geq 1}$ of rewriting polynomials involved in this rewriting trail; for $q \geq 1, A_{q}^{\prime} \in \mathbb{Z}[X], \operatorname{deg}\left(A_{q}^{\prime}\right) \leq q$ and $A_{q}^{\prime}(0)=-1$. The first polynomial $A_{1}^{\prime}(X)$ is $-1+\left(-a_{1}-t_{1}\right) X$. The second polynomial $A_{2}^{\prime}(X)$ is $-1+\left(-a_{1}-t_{1}\right) X+\left(-a_{2}-\right.$ $\left.\left(-\left(-a_{1}-t_{1}\right) t_{1}+t_{2}\right)\right) X^{2}$, etc.

For $q \geq \operatorname{deg}(P)$, all the coefficients of $P$ are restored; denote by $\left(h_{q, j}^{\prime}\right)_{j=0,1, \ldots, s-1}$ the $s$-tuple of integers produced by this rewriting trail, at step $q$. It is such that

$$
A_{q}^{\prime}\left(\gamma^{-1}\right) S_{\gamma}^{*}\left(\gamma^{-1}\right)=-P\left(\gamma^{-1}\right)+\gamma^{-q-1}\left(\sum_{j=0}^{s-1} h_{q, j} \gamma^{-j}\right) \text {. }
$$


Then take $q=d$. The lhs of (3.1.2) is equal to 0 . Thus

$$
P\left(\gamma^{-1}\right)=\gamma^{-d-1}\left(\sum_{j=0}^{s-1} h_{d, j} \gamma^{-j}\right) \quad \Longrightarrow \quad P(\gamma)=\sum_{j=0}^{s-1} h_{d, j} \gamma^{-j-1} .
$$

The height of the polynomial $W(X):=\sum_{j=0}^{s-1} h_{d, j} X^{j+1}$ is $\leq\left(2^{d}-1\right) H+2^{d}$. We now assume $|P(\gamma)|<\eta$. By Kala-Vavra's Theorem 1.1 there exist an alphabet $\mathscr{A} \subset \mathbb{Z}$, a preperiod $R(X) \in \mathscr{A}[X]$, a period $T(X) \in \mathscr{A}[X]$ such that

$$
W\left(\gamma^{-1}\right)=P(\gamma)=R\left(\gamma^{-1}\right)+\gamma^{-\operatorname{deg} R-1} \sum_{j=0}^{\infty} \frac{1}{\gamma^{j(\operatorname{deg} T+1)}} T\left(\gamma^{-1}\right)
$$

Since the relation $S_{\gamma}^{*}\left(\gamma^{-1}\right)=1-t_{1} \gamma^{-1}-t_{2} \gamma^{-2}-\ldots-t_{s-1} \gamma^{-s+1}-t_{s} \gamma^{-s}=0$ holds, we may assume $\operatorname{deg} R \leq s-1, \operatorname{deg} T \leq s-1$. Then, for $X$ specialized at $\gamma^{-1}$, we have the identity

$$
W(X)=R(X)+X^{L} \frac{T(X)}{1-X^{r}}
$$

for some positive integers $L, r$. The height of $\left(1-X^{r}\right) W(X)$ is $\leq 2\left(\left(2^{d}-1\right) H+2^{d}\right)$ and, with $\mathscr{A}$ assumed $=\{-m, \ldots, 0, \ldots,+m\}$, the height of $\left(1-X^{r}\right) R(X)+X^{L} T(X)$ is less than $3 m$. Therefore $m$ is $\leq 2\left(\left(2^{d}-1\right) H+2^{d}\right) / 3$. We can take $m=\left\lceil 2\left(\left(2^{d}-1\right) H+2^{d}\right) / 3\right\rceil$.

Since the algebraic norm $\mathrm{N}(\gamma)$ is equal to \pm 1 we cannot expect the uniqueness of the representations (3.1.3), for $X=\gamma^{-1}$, by [16]. However, for any $(\gamma, \mathscr{A})$-eventually periodic representation of $P(\gamma)$

$$
P(\gamma)=W\left(\gamma^{-1}\right)=\frac{a_{w}^{\prime}}{\gamma^{w}}+\frac{a_{w+1}^{\prime}}{\gamma^{w+1}}+\frac{a_{w+2}^{\prime}}{\gamma^{w+2}}+\ldots, \quad \text { with }\left|a_{j}^{\prime}\right| \leq m, j=w, w+1, \ldots
$$

with $a_{w}^{\prime} \neq 0$, the exponent $w$ appearing in the first term tends to infinity if $\eta$ tends to 0 . Indeed, from Theorem 4, Remarks 5 to 7, in [1], there exists a positive real number $\kappa_{\gamma, \mathscr{A}}>0$ such that $w$ is the minimal integer such that

$$
\gamma^{w-1} \geq \frac{\kappa_{\gamma, \mathscr{A}}}{|P(\gamma)|} \geq \frac{\kappa_{\gamma, \mathscr{A}}}{\eta}
$$

3.2. Application to Galois conjugation: convergence and eventually periodic representations along a sequence of almost Newman polynomials. Newman polynomials are polynomials with coefficients in $\{0,1\}$. In [5] almost Newman polynomials have been introduced: an almost Newman polynomial is an integer polynomial which has coefficients in $\{0,1\}$ except the constant term equal to -1 .

Definition 3.1. The collection of lacunary almost Newman polynomials of the type:

$$
f(x):=-1+x+x^{n}+x^{m_{1}}+x^{m_{2}}+\ldots+x^{m_{s}}
$$

where $n \geq 2, s \geq 0, m_{1}-n \geq n-1, m_{q+1}-m_{q} \geq n-1$ for $1 \leq q<s$, is called the class $\mathscr{B}$. The case " $s=0$ " corresponds to the trinomials $G_{n}(z):=-1+z+z^{n}$. The subclass $\mathscr{B}_{n} \subset \mathscr{B}$ is the set of polynomials $f(x) \in \mathscr{B}$ whose third monomial is exactly $x^{n}$, so that the union $\mathscr{B}=\cup_{n \geq 2} \mathscr{B}_{n}$ is disjoint.

The "Asymptotic Reducibility Conjecture", formulated in [5], says that 75\% of the polynomials $f(x) \in \mathscr{B}$ are irreducible. The factorization and the zeroes of the polynomials of the class $\mathscr{B}_{n}, n \geq 2$, have been studied in [5]. 
Theorem 3.2 (Selmer [24]). Let $n \geq 2$. The trinomials $G_{n}(x)$ are irreducible if $n \not \equiv 5(\bmod 6)$, and, for $n \equiv 5(\bmod 6)$, are reducible as product of two irreducible factors whose one is the cyclotomic factor $x^{2}-x+1$, the other factor $\left(-1+x+x^{n}\right) /\left(x^{2}-x+1\right)$ being nonreciprocal of degree $n-2$.

By definition, for $n \geq 2, \theta_{n}$ is the unique root of the trinomial $-1+x+x^{n}$ in the interval $(0,1)$. The algebraic integers $\theta_{n}^{-1}>1$ are Perron numbers. The sequence $\left(\theta_{n}^{-1}\right)_{n \geq 2}$ is decreasing, tends to 1 if $n$ tends to $+\infty$.

Theorem 3.3 (Dutykh - Verger-Gaugry [5]). For any $f \in \mathscr{B}_{n}, n \geq 3$, denote by

$$
f(x)=A(x) B(x) C(x)=-1+x+x^{n}+x^{m_{1}}+x^{m_{2}}+\ldots+x^{m_{s}},
$$

where $s \geq 1, m_{1}-n \geq n-1, m_{j+1}-m_{j} \geq n-1$ for $1 \leq j<s$, the factorization of $f$ where $A$ is the cyclotomic part, $B$ the reciprocal noncyclotomic part, $C$ the nonreciprocal part. Then

(i) the nonreciprocal part $C$ is nontrivial, irreducible and never vanishes on the unit circle,

(ii) if $\gamma>1$ denotes the real algebraic number uniquely determined by the sequence $\left(n, m_{1}, m_{2}, \ldots, m_{s}\right)$ such that $1 / \gamma$ is the unique real root of $f$ in $\left(\theta_{n-1}, \theta_{n}\right),-C^{*}(X)$ is the minimal polynomial $P_{\gamma}(X)$ of $\gamma$, and $\gamma$ is a nonreciprocal algebraic integer.

Now let us assume the existence of a reciprocal algebraic integer $\beta$ in the interval $\left(\theta_{n}^{-1}, \theta_{n-1}^{-1}\right)$ for some integer $n \geq 3$ ( $n$ is fixed), with $\mathrm{M}(\beta)<1.176280 \ldots$ Lehmer's number. It is canonically associated with, and characterized by, two analytic functions:

(i) its minimal polynomial, say $P_{\beta}$, which is monic and reciprocal meaning $X^{\operatorname{deg} P_{\beta}} P_{\beta}(1 / X)=P_{\beta}(X)$; denote $d:=\operatorname{deg} P_{\beta}, H:=$ the height of $P_{\beta}$; the minimal polynomial $P_{\beta}(X)$ of $\beta>1$ can be written

$$
P_{\beta}(X)=\widetilde{P_{\beta}}\left(X^{r}\right)
$$

for some integer $r \geq 1$ and some $\mathbb{Z}$-minimal integer polynomial $\widetilde{P_{\beta}}(X)$. The integer $r$ is the largest one such that (3.2.1) holds; it depends upon $\beta$. The $\beta$ s such that $r \geq 2$ are excluded in the following.

(ii) the Parry Upper function $f_{\beta}(x)$ at $\beta^{-1}$, which is the generalized Fredholm determinant of the $\beta$-transformation $T_{\beta}$ [2] which is a power series with coefficients in the alphabet $\{0,1\}$ except the constant term equal to -1 , with distanciation between the exponents of the monomials:

$$
f_{\beta}(x):=-1+x+x^{n}+x^{m_{1}}+x^{m_{2}}+\ldots+x^{m_{s}}+\ldots
$$

where $m_{1}-n \geq n-1, m_{q+1}-m_{q} \geq n-1$ for $q \geq 1$. $\beta^{-1}$ is the unique zero of $f_{\beta}(x)$ in the unit interval $(0,1)$. The analytic function $f_{\beta}(z)$ is related to the dynamical zeta function $\zeta_{\beta}(z)$ of the $\beta$-shift [11] [17] [21] by: $f_{\beta}(z)=-1 / \zeta_{\beta}(z)$. Since $\beta$ is reciprocal, with the two real roots $\beta$ and $1 / \beta$, the series $f_{\beta}(x)$ is never a polynomial, by Descartes's rule on sign changes on the coefficient vector. The algebraic integer $\beta$ is associated with the infinite sequence of exponents $\left(m_{j}\right)$. 
All the polynomial sections $S_{\gamma_{s}}^{*}(x):=-1+x+x^{n}+x^{m_{1}}+x^{m_{2}}+\ldots+x^{m_{s}}$ of $f_{\beta}(x)$ are polynomials of the class $\mathscr{B}_{n}$, mostly irreducible by the asymptotic reducibility conjecture, but not necessarily irreducible. For every $s \geq 1$, denote by $\gamma_{s}>1$ the (non-reciprocal) algebraic integer which is such that $\gamma_{s}^{-1}$ is the unique zero in $(0,1)$ of the polynomial section $S_{\gamma_{s}}^{*}(x)$ of $f_{\beta}(x)$. We have: $\operatorname{deg} \gamma_{s}^{-1}=\operatorname{deg} S_{\gamma_{s}}^{*}$ if and only if $S_{\gamma_{s}}^{*}(x)$ is irreducible. Moreover $f_{\beta}\left(\beta^{-1}\right)=0$ and $\lim _{s \rightarrow \infty} \gamma_{s}=\beta$.

We now apply Theorem 1.4:

The integer $n \geq 3$ is fixed. For all $s$ such that $\operatorname{deg} S_{\gamma_{s}}^{*} \geq \operatorname{deg} P_{\beta}$, the identity

$$
\mathbb{Q}\left(\gamma_{s}\right)=\operatorname{Per}_{\mathscr{A}}\left(\gamma_{s}\right),
$$

holds with $\mathscr{A}=\{-m, \ldots,+m\} \subset \mathbb{Z}, m=\left\lceil 2\left(\left(2^{d}-1\right) H+2^{d}\right) / 3\right\rceil$. By Theorem 3.3, for any $s \geq 0, \gamma_{s}^{-1}$ has no conjugate on the unit circle. The polynomial value $P_{\beta}\left(\gamma_{s}\right) \in \mathbb{Q}\left(\gamma_{s}\right)$ is eventually periodic

$$
P_{\beta}\left(\gamma_{s}\right)=R\left(\gamma_{s}^{-1}\right)+\frac{1}{\gamma_{s}^{L}} \sum_{j=0}^{\infty} \frac{1}{\gamma_{s}^{j \rho}} T\left(\gamma_{s}^{-1}\right) \quad \in \operatorname{Per}_{\mathscr{A}}\left(\gamma_{s}\right)
$$

with $L, \rho$ and $R(X), T(X)$, depending upon $s$. This representation of $P_{\beta}\left(\gamma_{s}\right)$ starts as

$$
=\frac{a_{w,(s)}}{\gamma_{s}^{w}}+\frac{a_{w+1,(s)}}{\gamma_{s}^{w+1}}+\ldots+\frac{a_{w+m_{s}-1,(s)}}{\gamma_{s}^{w+m_{s}-1}}+\ldots, \quad a_{j,(s)} \in \mathscr{A}, j=w, \ldots, w+m_{s}-1,
$$

with $a_{w,(s)} \neq 0$ and $w=w_{s} \geq 1$, depending upon $s$, satisfies $\frac{\kappa_{\gamma_{s}, \mathscr{A}}}{\eta} \leq \gamma_{s}^{w_{s}-1}$ for some positive constant $\kappa_{\gamma_{s}, \mathscr{A}}$ depending upon $\gamma$ and $\mathscr{A}$. Since $\mathscr{A}$ is independent of $s$, and that the sequence $\left(\gamma_{s}\right)$ is convergent with limit $\beta>1$, there exists a (true) constant $\kappa>0$ such that $\frac{\kappa}{\eta} \leq \gamma_{s}^{w_{s}-1}$ from Theorem 4, Remarks 5 to 7, in [1]. Since $\lim _{s \rightarrow \infty} P_{\beta}\left(\gamma_{s}\right)=0=P_{\beta}(\beta)$, we take $\eta=$ $\eta_{s}:=\left|P_{\beta}\left(\gamma_{s}\right)\right|$. The sequence $\left(\eta_{s}\right)$ tends to 0 . We deduce $\lim _{s \rightarrow \infty} w_{s}=+\infty$.

Let $\Omega \neq \beta^{-1}$ be a zero of modulus $<1$ of $f_{\beta}(x)$. We assume the existence of a small disk $D(\Omega, r)$ centered at $\Omega$ of radius $r>0$, included in the open unit disk, which has the property that the only zero of $f_{\beta}(x)$ in $D(\Omega, r)$ is $\Omega$. It is possible since the domain of existence of $f_{\beta}(x)$ is at least the open unit disk $D(0,1)$.

The zero $\Omega$ is limit point of a sequence of zeroes of the polynomial sections of $f_{\beta}(x)$. As soon as $s \geq s_{0}$ for some $s_{0}$, we assume that the disk $D(\Omega, r)$ contains only one zero of $S_{\gamma_{s}}^{*}(x)$. Denote by $r_{s}$ this zero. Let us assume that $r_{s}$ is a Galois conjugate of $\gamma_{s}^{-1}$, and denote by $\sigma_{s}: \gamma_{s}^{-1} \rightarrow r_{s}$ the $\mathbb{Q}$-automorphism of conjugation. This assumption is reasonable by the Asymptotic Reducibility Conjecture which says that $75 \%$ of the polynomial sections are irreducible.

The lenticular zeroes of $f_{\beta}$ are peculiar zeroes, off the unit circle. Let us briefly recall what is a lenticular zero of $f_{\beta}$. Many examples of lenticular zeroes are given in [5]. The following theorem is Theorem 4 in [5].

Theorem 3.4. Let $n \geq 260$. There exist two positive constants $c_{n}$ and $c_{A, n}, c_{A, n}<c_{n}$, such that the roots of any $f \in \mathscr{B}_{n}$,

$$
f(x)-1+x+x^{n}+x^{m_{1}}+x^{m_{2}}+\ldots+x^{m_{s}},
$$


where $s \geq 1, m_{1}-n \geq n-1, m_{j+1}-m_{j} \geq n-1$ for $1 \leq j<s$, lying in $-\pi / 18<\arg z<$ $+\pi / 18$ either belong to

$$
\left\{z \in \mathbb{C}|||z|-1 \mid<\frac{c_{A, n}}{n}\right\}, \quad \text { or to } \quad\left\{z \in \mathbb{C}|||z|-1 \mid \geq \frac{c_{n}}{n}\right\} .
$$

The lenticulus of zeroes $\omega$ of $f$ is then defined as

$$
\mathscr{L}_{\beta}:=\left\{\omega \in \mathbb{C}|f(\omega)=0,| \omega\left|<1,-\frac{\pi}{18}<\arg \omega<+\frac{\pi}{18},\right||\omega|-1 \mid \geq \frac{c_{n}}{n}\right\}
$$

where $1 / \beta \in \mathscr{L}_{\beta}$ is the positive real zero of $f$. If a zero of $f$ belongs to $\mathscr{L}_{\beta}$ we say that it is a lenticular zero of $f$.

Let us go back to the above assumption. If $\Omega$ is a lenticular zero of $f_{\beta}(x)$, then, by [5], all the polynomial sections $S_{\gamma_{s}}^{*}(x)$ do have also a (unique) lenticular zero close to $\Omega$ which is a conjugate of $\gamma_{s}^{-1}$. For the non-lenticular zeroes of $f_{\beta}(x)$, very close to the unit circle, the above assumption is necessary.

To summarize, for $s \geq s_{0}$ :

$$
f_{\beta}(\Omega)=0, \quad S_{\gamma_{s}}^{*}\left(r_{s}\right)=0, \quad r_{s}=\sigma_{s}\left(\gamma_{s}^{-1}\right), \quad\left|\Omega-\sigma_{s}\left(\gamma_{s}^{-1}\right)\right|<r, \quad \lim _{s \rightarrow \infty} r_{s}=\Omega .
$$

Let us show that $P_{\beta}(\Omega)=0$. Let us conjugate (3.1.3) for $X=\gamma_{s}^{-1}$. The power series (3.2.2) specialized at $\gamma_{s}^{-1}$ is eventually periodic, therefore can be conjugated term by term, once the image of $\gamma_{s}^{-1}$ by the conjugation $\sigma_{s}$ is such that $\left|\sigma_{s}\left(\gamma_{s}^{-1}\right)\right|<1$, to ensure convergence. Then

$$
\sigma_{s}\left(P_{\beta}\left(\gamma_{s}\right)\right)=W\left(r_{s}\right)=R\left(r_{s}\right)+r_{s}^{L} \frac{T\left(r_{s}\right)}{1-r_{s}^{\rho}}=a_{w_{s},(s)} r_{s}^{w_{s}}+a_{w_{s}+1,(s)} r_{s}^{w_{s}+1}+\ldots
$$

with

$$
\frac{\kappa}{\left|P_{\beta}\left(\gamma_{s}\right)\right|} \leq \gamma_{s}^{w_{s}-1} \quad \text { and } \quad\left|r_{s}\right|<|\Omega|+r<1, \quad s \geq s_{0}
$$

We have, with $m=\left\lceil 2\left(\left(2^{d}-1\right) H+2^{d}\right) / 3\right\rceil$,

$$
\begin{gathered}
\left|W\left(r_{s}\right)\right| \leq\left|a_{w_{s},(s)}\right|\left|r_{s}^{w_{s}}\right|+\left|a_{w_{s}+1,(s)}\right|\left|r_{s}^{w_{s}+1}\right|+\ldots \\
\leq m\left(\left|r_{s}^{w_{s}}\right|+\left|r_{s}^{w_{s}+1}\right|+\ldots\right)=m\left|r_{s}^{w_{s}}\right|\left(1+\left|r_{s}\right|+\left|r_{s}\right|^{2}+\ldots\right) .
\end{gathered}
$$

Then

$$
\left|W\left(r_{s}\right)\right| \leq\left|r_{s}\right|^{w_{s}} \frac{m}{1-\left|r_{s}\right|} .
$$

Let us observe that within a period of period length $\rho$ in the power series (3.2.3) a certain number of coefficients are equal to zero, and therefore that the upper bound (3.2.4) can be improved using the period length $\rho$ and the degree of $T$. However it is sufficient for below. We deduce

$$
P_{\beta}(\Omega)=\lim _{s \rightarrow \infty} W\left(r_{s}\right)=0 .
$$

Under the above assumptions, we have proved:

Proposition 3.5.

$$
f_{\beta}(\Omega)=0 \quad \Longrightarrow \quad P_{\beta}(\Omega)=0 .
$$


Remark 3.6. As a consequence the properties of the analytic function

$$
f_{\beta}(x):=-1+x+x^{n}+x^{m_{1}}+x^{m_{2}}+\ldots+x^{m_{s}}+\ldots, \quad|x|<1,
$$

where $m_{1}-n \geq n-1, m_{q+1}-m_{q} \geq n-1$ for $q \geq 1$ can be used to investigate the geometry of the zeroes of the polynomial $P_{\beta}(X)$, in particular the existence of integer polynomials having a very small Mahler measure below Lehmer's number $1.176280 \ldots$.. Let us note that the Parry Upper function $f_{\beta}(z)$ at $\beta$ (here reciprocal) is related to the dynamical zeta function $\zeta_{\beta}(z)$ of the $\beta$-shift by: $f_{\beta}(z)=-1 / \zeta_{\beta}(z)$ ([17]; see Solomyak [25] for the zeroes). In this respect, Proposition 3.5 says that the poles of modulus $<1$ of the meromorphic extension of $\zeta_{\beta}(z)$ in the open unit disk are, under some assumptions (as mentioned above), zeroes of the minimal polynomial of $\beta$.

\subsection{Natural and intermediate alphabets along sequences of almost Newman polyno-} mials: examples. When the base $\gamma$ is fixed, as in Theorem 1.4, the intermediate alphabet produced by a rewriting trail has a size growing linearly with the height $H$ of the polynomial $P$. Leaving $\gamma$ fixed and varying $H$ in $P$, when $H$ becomes very large, this intermediate alphabet reaches the natural alphabet $\mathscr{A}_{\gamma}^{(\text {nat })}$, becomes equal to it, exceeds it; so that there is no interest to proceed with rewriting trails for such polynomials having a large height, i.e. for $H$ above a certain critical value.

The natural alphabets $\mathscr{A}_{\gamma_{s}}^{(\text {nat })}$ along the sequence of the polynomial sections $S_{\gamma_{j}}^{*}(X)=$ $-1+x+x^{n}+x^{m_{1}}+x^{m_{2}}+\ldots+x^{m_{j}}, j \geq 1$, of a given Parry Upper function

$$
f_{\beta}(z)=-1+x+x^{n}+x^{m_{1}}+x^{m_{2}}+\ldots+x^{m_{j}}+\ldots
$$

i.e. along a sequence of bases $\left(\gamma_{s}\right)$, as in Section 3.2, are huge and do not remain constant. Let us take examples. The following alphabets $\mathscr{A}_{\gamma_{j}}^{(\text {nat })}=\left\{-m_{\gamma_{j}}, \ldots,+m_{\gamma_{j}}\right\}$ are calculated by (2.0.1) and Proposition 2.1. Denote $m=m_{\gamma_{j}}$ for short. The integer $j$ is the number of monomials added to the trinomial $-1+x+x^{11}$. All the polynomials are irreducible, of the same degree $(=101)$, and belong to the class $\mathscr{B}_{11}$ :

$\mathrm{j}=1: \mathrm{p}:=x^{101}+x^{11}+x-1$,

$m=2.617526038 * 10^{365}$

$\mathrm{j}=2: \mathrm{p}:=x^{101}+x^{21}+x^{11}+x-1$,

$m=4.088496786 * 10^{288}$;

$\mathrm{j}=3: \mathrm{p}:=x^{101}+x^{35}+x^{21}+x^{11}+x-1$,

$m=3.196582086 * 10^{151}$;

$\mathrm{j}=4: \mathrm{p}:=x^{101}+x^{45}+x^{35}+x^{21}+x^{11}+x-1$,

$m=3.823048784 * 10^{462}$;

$\mathrm{j}=5: \mathrm{p}:=x^{101}+x^{57}+x^{45}+x^{35}+x^{21}+x^{11}+x-1$,

$m=8.866692051 * 10^{248}$;

$\mathrm{j}=6: \mathrm{p}:=x^{101}+x^{69}+x^{57}+x^{45}+x^{35}+x^{21}+x^{11}+x-1$,

$m=4.851172757 * 10^{224}$;

$\mathrm{j}=7: \mathrm{p}:=x^{101}+x^{80}+x^{69}+x^{57}+x^{45}+x^{35}+x^{21}+x^{11}+x-1$,

$m=6.062823380 * 10^{222}$; 


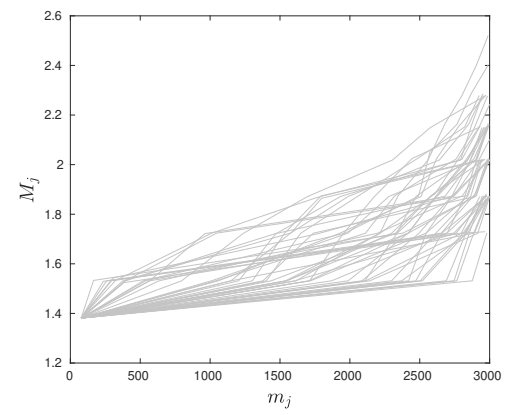

FIGURE 2. Mahler measures $\mathrm{M}_{j}=\mathrm{M}\left(S_{\gamma_{j}}^{*}\right)$ of $j$ th-polynomial sections $S_{\gamma_{j}}^{*}$ of $f_{\beta}(z)$ for various $\theta_{77}^{-1}<\beta<\theta_{76}^{-1}$, as a function of the degree $m_{j}:=$ $\operatorname{deg} S_{\gamma_{j}}^{*}$. The initial value is $\mathrm{M}_{0}=\mathrm{M}\left(-1+x+x^{77}\right) \approx 1.38$ by [9] [29].

$$
\begin{aligned}
& \mathrm{j}=7: \mathrm{p}:=x^{101}+x^{81}+x^{69}+x^{57}+x^{45}+x^{35}+x^{21}+x^{11}+x-1, \\
& m=4.617819094 * 10^{1083} ; \\
& \mathrm{j}=8: \mathrm{p}:=x^{101}+x^{91}+x^{80}+x^{69}+x^{57}+x^{45}+x^{35}+x^{21}+x^{11}+x-1, \\
& m=2.085371358 * 10^{536} ; \\
& \mathrm{j}=8: \mathrm{p}:=x^{101}+x^{90}+x^{80}+x^{69}+x^{57}+x^{45}+x^{35}+x^{21}+x^{11}+x-1, \\
& m=3.484819567 * 10^{196}
\end{aligned}
$$

No simple law of $m_{\gamma_{j}}$ appears as a function of $j$ : for $j=7$, resp. $j=8$, a big difference appear in the size of the alphabets, obtained by varying just one monomial in the definition of $\mathrm{p}$.

On the contrary, the alphabets obtained by rewriting trails along the sequence of the polynomial sections $S_{\gamma_{j}}^{*}(X)$, from a given polynomial, remain constant.

Given $f_{\beta}(x)$ as in (3.3.1), the growth rate of the natural alphabets $\mathscr{A}_{\gamma_{j}}^{(\text {nat })}$ with the degree $m_{j}$ and the number $j$ of monomials is investigated, in the Figures 2 to 7 , in terms of the Mahler measures

$$
\mathbf{M}\left(S_{\gamma_{j}}^{*}\right)=\mathbf{M}\left(-1+x+x^{n}+x^{m_{1}}+x^{m_{2}}+\ldots+x^{m_{j}}\right)
$$

this is sufficient according to the approximate lower bounds

$$
\frac{1}{2} \mathrm{M}\left(\gamma_{j}\right)^{N_{j}-1}\left|S_{\gamma_{j}}^{*}(1)\right|=\frac{j+1}{2} \mathrm{M}\left(\gamma_{j}\right)^{N_{j}-1}
$$

by (2.1.5), where the $j$-th integer $N_{j}$ is the dominance index relative to $\gamma_{j}$, and that the identities $\mathrm{M}\left(S_{\gamma_{j}}^{*}\right)=\mathrm{M}\left(\gamma_{j}\right), S_{\gamma_{j}}(X)=P_{\gamma_{j}}(X)$, hold for $75 \%$ of the polynomial sections, by the Asymptotic Reducibility Conjecture. Mahler measures are calculated by means of Graeffe's method [10] and in PARI/GP [26]. The Mahler measure $\mathrm{M}\left(S_{\gamma_{j}}^{*}\right)$ is a function of the geometry of the roots of $S_{\gamma_{j}}^{*}(X)$ which lie inside the open unit disk; the respective roles of the non-lenticular roots with respect to the lenticular roots [5] are investigated in [6]. The fairly large values $N_{j}$ of the dominance indices, arising from the arithmetics of the iteration of the companion matrix of $P_{\beta}$, are not indicated. The values $N_{j}$ are related to the dynamical system with polynomial action, see Remark 2.5 . 


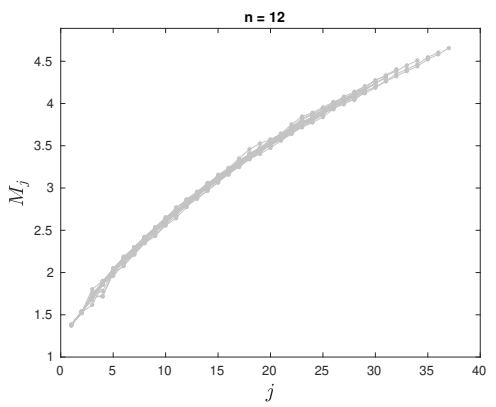

FIGURE 3. Mahler measures $\mathrm{M}_{j}=\mathrm{M}\left(S_{\gamma_{j}}^{*}\right)$ of $j$ th-polynomial sections $S_{\gamma_{j}}^{*}$ of $f_{\beta}(z)$ for various $\theta_{12}^{-1}<\beta<\theta_{11}^{-1}$, as a function of the number $j$ of monomials added to $-1+x+x^{12}$. The initial value is $\mathrm{M}\left(-1+x+x^{12}\right) \approx 1.38$ by [9] [29].

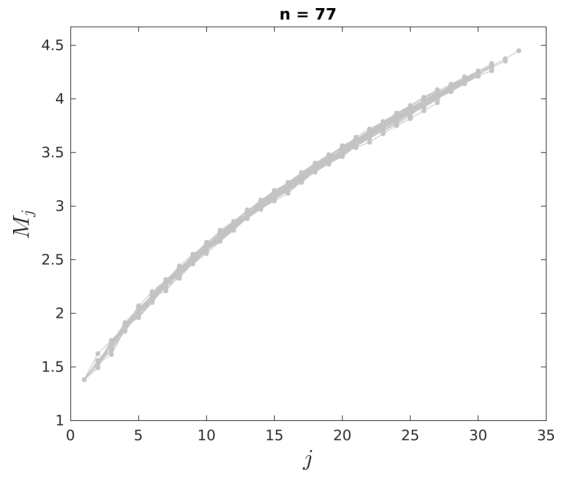

FIGURE 4. Mahler measures $\mathrm{M}_{j}=\mathrm{M}\left(S_{\gamma_{j}}^{*}\right)$ of $j$ th-polynomial sections $S_{\gamma_{j}}^{*}$ of $f_{\beta}(z)$ for various $\theta_{77}^{-1}<\beta<\theta_{76}^{-1}$, as a function of the number $j$ of monomials added to $-1+x+x^{77}$. The initial value is $\mathrm{M}\left(-1+x+x^{77}\right) \approx 1.38$ by [9] [29].

Along the sequence of the polynomial sections $S_{\gamma_{j}}^{*}(X)$ of $f_{\beta}(z)$, for $\theta_{n}^{-1}<\beta<\theta_{n-1}^{-1}$, $n \geq 3$, any algebraic integer, the sequence of the exponents $\left(m_{j}\right)$ satisfies

$$
1+\frac{n-1}{m_{j}} \leq \frac{m_{j+1}}{m_{j}}, \quad \limsup _{j \rightarrow \infty} \frac{m_{j+1}}{m_{j}} \leq \frac{\log (\mathrm{M}(\beta))}{\log \beta}
$$

by Theorem 1.1 in [28], so that the lacunarity in $f_{\beta}(z)$ remains moderate, and the number of monomials in a section $S_{\gamma_{j}}^{*}(X)$ is always increasing with $j$ with positive density. The topo$\operatorname{logical}$ entropies $\log \mathrm{M}(\beta)$ and $\log \beta$ are related to the two dynamical systems involved in the sequences of coefficients of $f_{\beta}(z)$, see Remark 2.5 and [28].

In Figure 3 to Figure 6 the Mahler measures $\mathrm{M}_{j}=\mathrm{M}\left(S_{\gamma_{j}}^{*}\right)$ of the $j$ th-polynomial sections $S_{\gamma_{j}}^{*}$ of $f_{\beta}(z)$ are represented for various $\theta_{n}^{-1}<\beta<\theta_{n-1}^{-1}$, as a function of the number $j$ of monomials added to $-1+x+x^{n}$, for different values of $n: n=12,77,149,220$. The initial value is $\mathrm{M}\left(-1+x+x^{n}\right) \approx 1.38$ by [9], [29]. The growth rates are close to obey a linear growth with $j$. Each time, the growth of $\mathbf{M}_{j}$ occurs with $j$, without stabilization except in 


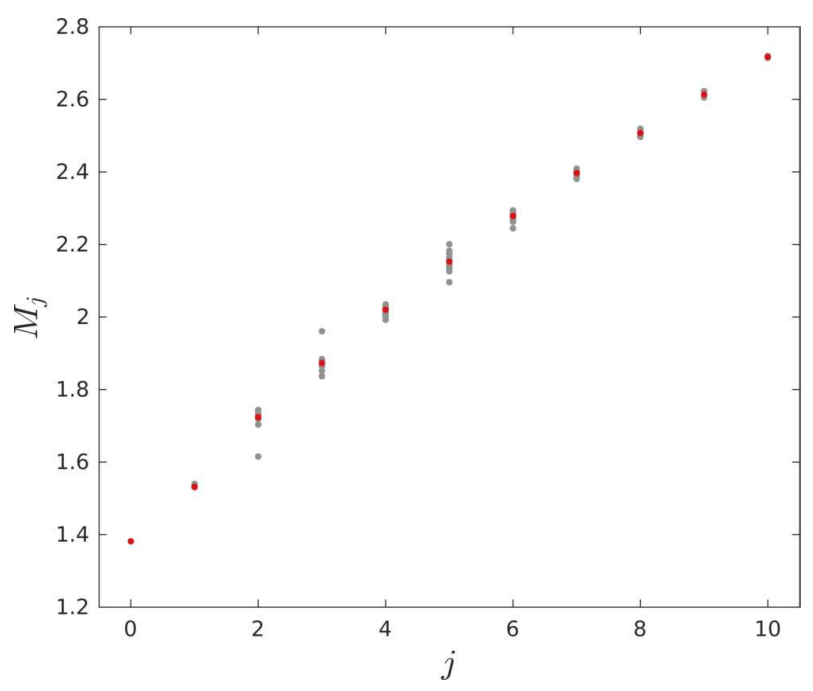

FIGURE 5. Mahler measures $\mathrm{M}_{j}=\mathrm{M}\left(S_{\gamma_{j}}^{*}\right)$ of $j$ th-polynomial sections $S_{\gamma_{j}}^{*}$ of $f_{\beta}(z)$ for various $\theta_{149}^{-1}<\beta<\theta_{148}^{-1}$, as a function of the number $j$ of monomials added to $-1+x+x^{149}$. The initial value is $\mathrm{M}\left(-1+x+x^{149}\right) \approx 1.38$ by [9] [29].

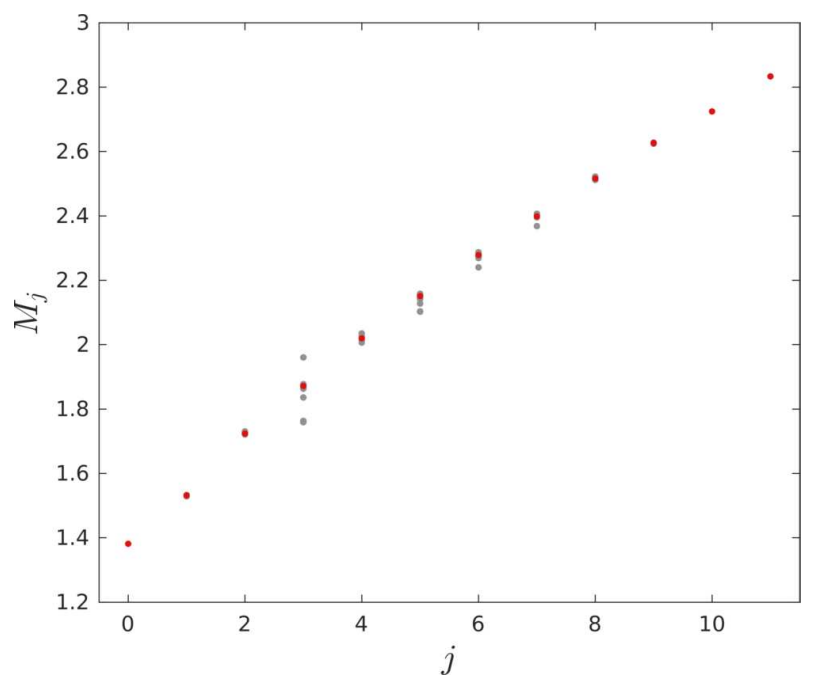

FIGURE 6. Mahler measures $\mathrm{M}_{j}=\mathrm{M}\left(S_{\gamma_{j}}^{*}\right)$ of $j$ th-polynomial sections $S_{\gamma_{j}}^{*}$ of $f_{\beta}(z)$ for various $\theta_{220}^{-1}<\beta<\theta_{219}^{-1}$, as a function of the number $j$ of monomials added to $-1+x+x^{220}$. The initial value is $\mathrm{M}\left(-1+x+x^{220}\right) \approx 1.38$ by [9] [29].

Figure 7 where a plateau appears when the sequence of exponents $\left(m_{j}\right)$ is purely periodic (with period length $n-1$ ). 


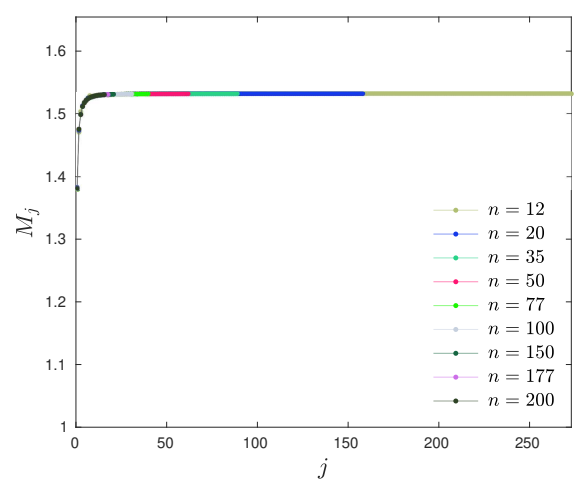

FIGURE 7. Minimal lacunarity: Mahler measure $\mathbf{M}_{j}=\mathbf{M}\left(S_{\gamma_{j}}^{*}\right)$ of the eventually periodic section $S_{\gamma_{j}}^{*}(x)=-1+x+x^{n}+x^{m_{1}}+\ldots+x^{m_{j}}, m_{1}-n=n-1$, $m_{q+1}-m_{q}=n-1$ for $q \geq 1$, as a function of the number of monomials $j$ added to the trinomial $-1+x+x^{n}$, for various values of $n$. The initial value is $\mathrm{M}\left(-1+x+x^{n}\right) \approx 1.38$ by [9] [29]. The increase of $\mathrm{M}_{j}$ is followed by a plateau.

\section{ACKNOWLEDGEMENTS}

We would like to thank the anonymous referee for his helpful comments.

\section{REFERENCES}

[1] S. Baker, Z. Masáková, E. Pelantová and T. VÁvra, On Periodic Representations in non-Pisot Bases, Monatsh. Math. 184 (2017), 1-19.

[2] V. BALADI and Keller, Zeta Functions and Transfer Operators for Piecewise Monotone Transformations, Comm. Math. Phys. 127 (1990), 459-477.

[3] M.-J. Bertin, A. Decomps-Guilloux, M. Grandet-Hugot, M. PathiauX-Delefosse and J.P. SChreIBER, Pisot and Salem Numbers, Birkhäuser Verlag (1992).

[4] Z. DARóCZY and I. KÁtAi, Generalized Number Systems in the Complex Plane, Acta Math. Hungar. 51 (1988), 409-416.

[5] D. Dutykh and J.-L. Verger-Gaugry, On the Reductibility and the Lenticular Sets of Zeroes of Almost Newman Lacunary Polynomials, Arnold Math. J. 4 (2018), 315-344.

[6] D. Dutykh and J.-L. Verger-Gaugry, Spirals of Poles of the Dynamical Zeta Function of the $\beta$-shift for $\beta$ close to one, and Lehmer's Problem, preprint (2021).

[7] M. Einsiedler, G. Everest and T. WARD, Primes in Sequences Associated to Polynomials (after Lehmer), LMS. J. Comput. Math. 3 (2000), 125-139.

[8] G. Everest and T. WARD, Heights of Polynomials and Entropy in Algebraic Dynamics, SpringerVerlag, 1999.

[9] V. Flammang, The Mahler Measure of Trinomials of Height 1, J. Austral. Math. Soc 96 (2014), 231243.

[10] V. Flammang, G. Rhin and J.-M. SaC-Epée, Integer Transfinite Diameter and Polynomials with Small Mahler Measure, Math. Comp. 75:255 (2006), 1527-1540.

[11] C. Frougny, Chap. 7 "Numeration Systems" in [21].

[12] C. Frougny, E. Pelantová and M. Svobodová, Parallel Addition in Non-standard Numeration Systems, Theor. Comput. Sci. 412 (2011), 5714-5727.

[13] V. Kala and T. VÁvra, Periodic Representations in Algebraic Bases, Monatsh. Math. 188 (2019), 109-119. 
[14] H. Kestelman, Automorphisms of the field of complex numbers, Proc. London Math. Soc. 53 (1951), $1-12$.

[15] B. Kovács, Canonical Number Systems in Algebraic Number Fields, Acta Math. Hungar. 37 (1981), 405-407.

[16] B. KovÁcs and I. KöRnYeI, On the Periodicity of the Radix Expansion, Ann. Univ. Sci. Budapest. Sect. Comput. 13 (1992), 129-133.

[17] J.C. Lagarias, Number Theory Zeta Functions and Dynamical Zeta Functions, in Spectral problems in Geometry and Arithmetic, Contemp.Math., 237, Amer. Math. Soc., Providence, RI (1999), 45-86.

[18] P. LanCAster, Theory of Matrices, Academic Press (1969).

[19] D.H. LeHMER, Factorization of Certain Cyclotomic Functions, Ann. of Math. 34 (1933), 461-479.

[20] D.A. Lind , Dynamical Properties of Quasihyperbolic Toral Automorphisms, Ergodic Theory Dynam. Systems 2 (1982), 49-68.

[21] M. Lothaire, Algebraic Combinatorics on Words, Cambridge University Press, Cambridge (2002).

[22] A. Рethő, On the Periodic Expansion of Algebraic Numbers, Ann. Univ. Sci. Budapest. Sect. Comput. 18 (1999), 167-174.

[23] K. Schmidt, On Periodic Expansions of Pisot Numbers and Salem Numbers, Bull. London Math. Soc. 12 (1980), 269-278.

[24] E.S. Selmer, On the Irreducibility of Certain Trinomials, Math. Scand. 4 (1956), 287-302.

[25] B. SOLOMYAK, Conjugates of beta-numbers and the zero-free domain for a class of analytic functions, Proc. London Math. Soc. 68 (1994), 477-498.

[26] THE PARI GROUP, PARI/GP version 2.11 .2 (2019), "Univ. Bordeaux", available from http://pari.math.u-bordeaux.fr/

[27] W.P. ThURston, Groups, Tilings and Finite State Automata: Summer 1989 AMS Colloquium Lectures, Research Report GCG, Geometry Computing Group, 1989.

[28] J.-L. Verger-Gaugry, On Gaps in Rényi $\beta$-expansions of Unity for $\beta>1$ an Algebraic Number, Annales Inst. Fourier Grenoble 56 (2006), 2565-2579.

[29] J.-L. Verger-Gaugry, On the Conjecture of Lehmer, Limit Mahler Measure of Trinomials and Asymptotic Expansions, Uniform Distribution Theory J. 11 (2016), 79-139.

[30] P.B. YALES Automorphisms of the Complex Numbers, Math. Mag. 39 (1966), 135-141.

†Univ. Grenoble Alpes, Univ. Savoie Mont Blanc, CNRS UMr 5127, LAMA, F-73000 CHAMBÉRY, FrANCE

Email address: Denys. Dutykh@univ-smb.fr

¥Univ. Grenoble Alpes, Univ. Savoie Mont Blanc, CNRS UMr 5127, LAMA, F-73000 CHAMBÉRY, FRANCE

Email address: Jean-Louis.Verger-Gaugry@univ-smb.fr 